\title{
Risk factors for necrotising enterocolitis: the influence of gestational age
}

\author{
Philip J Beeby, Heather Jeffery
}

\begin{abstract}
Over a 7 year study period, 82 infants were identified who had necrotising enterocolitis (NEC). A case-control study of the 74 preterm infants was performed to determine those factors which contributed to the development of NEC. The 35 infants with NEC and gestation between 30-36 weeks, when compared with control infants matched for gestational age, had significantly lower birthweight centiles, cord pH, and 1 minute Apgar scores. By contrast, there were no significant differences between the 39 infants with NEC and controls in the 25-29 week group, except that fewer babies with NEC had received breast milk. The eight term babies all appeared to have an obvious predisposing event. We thus propose a model in which susceptibility to NEC is dependent on gestational age. In the 25-29 week range all babies are at risk on the basis of extreme prematurity. In the 30-36 week range asphyxiated and growth retarded babies are at increased risk, while at term a major predisposing event appears to be required.
\end{abstract}

Necrotising enterocolitis (NEC) remains a major life threatening disease seen in neonatal intensive care units, and despite investigation by many groups the aetiology of the disease has defied clear explanation. A number of groups have suggested that for preterm infants, prematurity is the only perinatal factor that is related to an increased risk of NEC. ${ }^{1-5}$ However, in other reports in which risk factors for NEC were identified, these often differed from study to study. For example, hyaline membrane disease was reportedly more common in patients with NEC, ${ }^{6-8}$ or was more common in controls. ${ }^{14}$ Similarly, in some reports asphyxia ${ }^{7-10}$ and umbilical artery catheterisation ${ }^{6-8} 10$ were associated with NEC, but were not in others. ${ }^{1-5} 9$ il The most likely explanation for this lack of agreement lies in the differences in patient populations in the studies. Those studies examining the most premature babies $(<1500$ $\mathrm{g}^{4}{ }^{11}<1750 \mathrm{~g}^{3}$ ) tended to report a lack of NEC risk factors, while those which included all newborns up to term tended to report positive findings. Furthermore, there were small numbers of cases of NEC in some studies, different management policies, and the inclusion of varying numbers of outborn babies in some series.

There is mounting evidence that risk factors vary with birth weight or gestational age. ${ }^{79} 12$ In those studies utilising case-control design, matching for birth weight was most commonly employed. ${ }^{12} 581011$ Such a choice is less than ideal as it fails to take into account the large differences in maturity that occur between small for dates and large for dates babies of similar weight. One study controlled for gestational age, but this was confined to 'late onset' cases of NEC and included only 23 cases. $^{13}$

Recent reports have provided new information on the aetiology of NEC by demonstrating a highly significant association between absent or reversed end diastolic flow velocity waveforms (AREDF) in the umbilical artery or fetal aorta and the subsequent development of NEC. ${ }^{14}$ is For the first time a subgroup of preterm infants has been identified which appears to be at greatly increased risk of developing NEC. Although, such infants are on average very growth retarded, Malcolm et al, in a casecontrol study in which infants were matched for gestational age and birth weight, concluded that the risk of NEC was related to the AREDF rather than growth retardation. ${ }^{14}$ Nevertheless, one might anticipate an increased incidence of NEC among growth retarded infants, and it is surprising that such an association has not been reported in the many previous studies involving the identification of risk factors for NEC.

We were prompted to undertake a study of NEC at King George V Hospital for Women because of the persisting occurrence of NEC, despite significant advances in other areas of neonatal management. We decided to undertake a case-control study, preferring to control for gestational age for the reasons mentioned above.

\section{Subjects and methods}

All newborn infants born between January 1984 and March 1991 who developed NEC were included in this retrospective study. King George V Hospital for Women is a teaching hospital for the University of Sydney with about 4500 deliveries annually. Although mainly drawing upon the surrounding inner city areas, it is also a major referral centre for the in utero transfer of high risk pregnancies, with a large proportion of the babies $<32$ weeks' gestation $(45 \%)$ being in this category.

NEC was defined as the presence of clinical features of NEC (bilious aspirates or vomits, abdominal distension, systemic features such as lethargy and apnoea, with or without bloody stools) and radiological features (pneumatosis coli with or without gas in the portal circulation), and/or pathological evidence from laparotomy or necropsy specimens. Pneumoperitoneum 
without pneumatosis was not taken to indicate NEC unle'ss there was unequivocal evidence from pathological specimens, as spontaneous perforation of the gut is a well recognised entity that appears to be distinct from NEC. ${ }^{16}$ Using these strict guidelines, 82 cases were identified.

Control infants were selected from the admission book of the neonatal intensive care unit and were defined as the infant born nearest in time and with the same gestational age to that of the infant with NEC. The only grounds for rejecting a control were failure to survive for the number of days equal to age at onset of NEC in the matched infant. In the majority of cases an early ultrasound result was available to confirm the gestational age, which was nominated from the antenatal data on day 1 . Birthweight centiles were calculated using standard methods ${ }^{17}$ and were derived from birth weight and gestational age data at this hospital over the period of the study.

Most of the data required for this study were obtained from the computerised database of the neonatal intensive care unit, into which data for babies admitted to the nursery had been routinely entered since the beginning of the study period. Cases of NEC were then checked against manual records kept over the study period. Recall of medical records was necessary to obtain details of infant feeding which had not been included in the unit database.

For analysis of matched case-control pairs, SPSS/PC $+{ }^{18}$ and EPI-INFO ${ }^{19}$ computer statistical programs were employed. McNemar's test was used for comparing dichotomous variables. On testing of continuous variables, only birthweight centile was normally distributed, and paired $t$ tests were employed. Wilcoxon's signed ranks test was used for comparison of the other continuous variables.

\section{Results}

\section{GENERAL FEATURES}

Eighty two cases of NEC were identified over the 87 month study period, representing an incidence of 9.4 cases/year. The mean birth weight was $1418 \mathrm{~g}$ (mean birthweight centile $37 \%$ ) and the mean gestational age 30.4 weeks. There were 23 deaths, giving an overall mortality rate of $28 \%$. The mean gestational age of infants who died was 28 weeks, with the oldest being 34 weeks, whereas the mean for surviving babies was 31 weeks. Overall, 30 babies (37\%) underwent surgery of whom $16(53 \%)$ were survivors. Of the blood cultures collected on diagnosis of the NEC, 20 (24\%) were positive, with the following pattern: Klebsiella spp $(\mathrm{n}=7)$, Staphylococcus epidermidis $(n=6)$, Escherichia coli $(\mathbf{n}=3)$, Clostridium spp $(\mathbf{n}=2)$, Enterobacter spp $(n=1)$, and $\alpha$-haemolytic streptococcus $(n=1)$.

In all cases of NEC the baby had been fed before the onset of symptoms. The age at onset of NEC overall had a mean of 11 days, but with a mode of 5 and median of 9 . There was a pattern of early onset NEC with the older babies and late onset NEC with the more premature. There was a significant negative correlation between gestational age and day of onset of
NEC $(r=-0.35, p=0.002)$, in agreement with previous studies. ${ }^{2}$

The 82 babies included 74 preterm and eight term infants. Because the term babies appeared to have obvious predisposing factors they were analysed separately from the preterm infants.

\section{PRETERM INFANTS}

The incidence of NEC in each gestational age category is presented in fig 1 . Included in fig 1 is the number of babies with NEC at each gestational age. The mean birthweight centiles for babies with NEC and for controls at each gestational age are presented in fig 2. Overall, the babies with NEC had significantly lower birth weights than the controls $(p<0.001)$. They were significantly more growth retarded, as evidenced by the lower mean birthweight centiles $(p<0.001)$, or the higher proportion of infants less than the 10th centile $(p=0.01$, odds ratio (OR) $3 \cdot 6,95 \%$ confidence interval $(\mathrm{CI}) 1 \cdot 3$ to $9 \cdot 7)$.

INFANTS < 30 WEEKS' GESTATION

The differences between NEC and controls for birthweight centile were most noticeable for infants of 30-36 weeks' gestation, whereas in those of 25-29 weeks the corresponding differences appeared to be less significant. In order to highlight this important result, analysis was performed separately for babies of 25-29 and 30-36 weeks' gestation. The results for the 39 cases in the 25-29 week group' are presented in table 1 .

The striking result that emerged was the lack of identifiable risk factors in this age category,

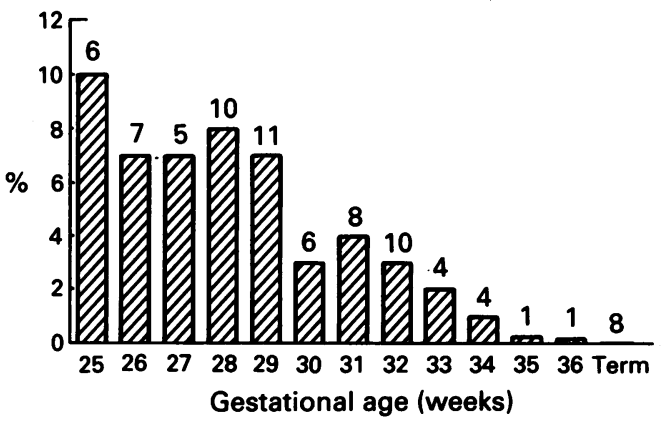

Figure 1 Incidence (\%) of NEC by gestational age, including total number with NEC at each gestational age.

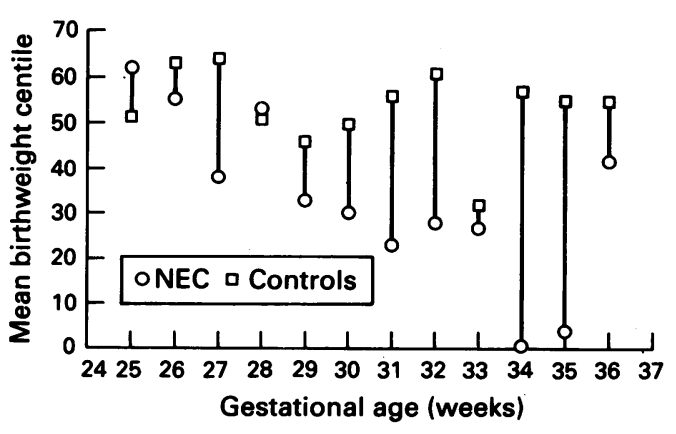

Figure 2 Mean birthweight centiles for babies with NEC and for controls. 
apart from increased mortality in the NEC infants. However, factors that did reach significance were the higher proportion of babies who received infant formula alone in the NEC group $(p=0.04, O R=4 \cdot 0, C I=1 \cdot 1$ to $14 \cdot 1)$, and the lower proportion of babies who received only expressed breast milk in the NEC group $(\mathrm{p}=0.03, \mathrm{OR}=0.29, \mathrm{CI}=0.09$ to 0.87$)$.

INFANTS 30-36 WEEKS' GESTATION

The results for the 35 infants in this category together with their controls is presented in table 2. The birth weights and birthweight centiles for the babies with NEC were significantly

Table 1 Comparison of clinical variables in case-control matched pairs 25-29 weeks' gestation. Results are given as percentages of babies with factor present or as means for continuous variables

\begin{tabular}{|c|c|c|c|}
\hline Clinical variable & $N E C$ & Controls & Comparison \\
\hline 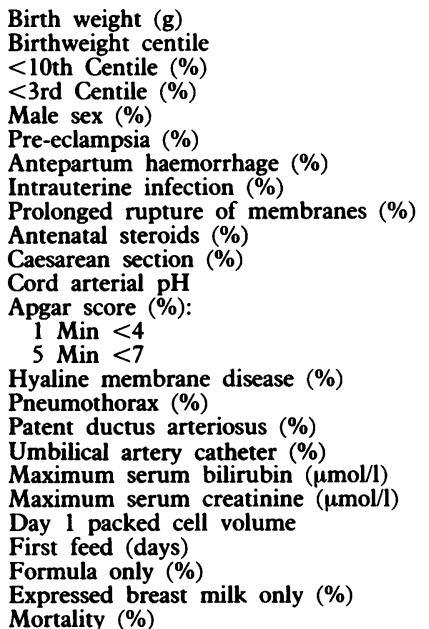 & $\begin{array}{l}1013 \\
47 \\
15 \\
5 \\
39 \\
28 \\
31 \\
39 \\
36 \\
72 \\
54 \\
7 \cdot 21 \\
39 \\
33 \\
67 \\
15 \\
41 \\
33 \\
205 \\
130 \\
0 \cdot 45 \\
4 \cdot 7 \\
39 \\
13 \\
41\end{array}$ & $\begin{array}{l}1052 \\
53 \\
8 \\
5 \\
41 \\
15 \\
21 \\
49 \\
49 \\
80 \\
36 \\
7 \cdot 23 \\
33 \\
23 \\
44 \\
26 \\
39 \\
18 \\
216 \\
125 \\
0 \cdot 45 \\
4 \cdot 5 \\
15 \\
39 \\
5\end{array}$ & 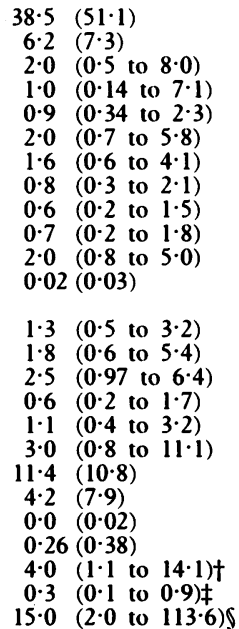 \\
\hline
\end{tabular}

"For continuous variables the mean difference and standard error are presented and for dichotomous variables the odds ratio and $95 \%$ confidence intervals are given. p Values were obtained using McNemar's test for the dichotomous variables, paired $t$ test for birthweight centile data, and Wilcoxon rank sign test for other continuous variables.

$\mathrm{tp}=0.04, \neq p=0 \cdot 03,\lceil p=0 \cdot 001$

Table 2 Comparison of clinical variables in 35 case-control matched pairs of infants 30-36 weeks' gestation. Results given as percentage of babies with factor present or as means for continuous variables

\begin{tabular}{|c|c|c|c|}
\hline Clinical variable & $N E C$ & Controls & Comparison: \\
\hline $\begin{array}{l}\text { Birth weight (g) } \\
\text { Birthweight centile } \\
\text { <10th Centile (\%) } \\
\text { <3rd Centile (\%) } \\
\text { Male sex (\%) } \\
\text { Pre-eclampsia (\%) } \\
\text { Antepartum haemorrhage (\%) } \\
\text { Intrauterine infection (\%) } \\
\text { Prolonged rupture of membranes (\%) } \\
\text { Antenatal steroids (\%) } \\
\text { Caesarean section (\%) } \\
\text { Cord arterial pH } \\
\text { Apgar score }(\%) \text { : } \\
1 \text { Min <4 } \\
5 \text { Min <7 } \\
\text { Hyaline membrane disease (\%) } \\
\text { Pneumothorax (\%) } \\
\text { Patent ductus arteriosus (\%) } \\
\text { Umbilical artery catheter (\%) } \\
\text { Maximum serum bilirubin ( } \mu \text { mol/l) } \\
\text { Maximum serum creatinine ( }(\mu \mathrm{mol} / \mathrm{l}) \\
\text { Day } 1 \text { packed cell volume } \\
\text { First feed (days) } \\
\text { Formula only (\%) } \\
\text { Expressed breast milk only (\%) } \\
\text { Mortality (\%) }\end{array}$ & $\begin{array}{l}1479 \\
24 \\
37 \\
29 \\
60 \\
34 \\
17 \\
11 \\
6 \\
66 \\
77 \\
7 \cdot 21 \\
31 \\
20 \\
43 \\
6 \\
9 \\
11 \\
225 \\
112 \\
0 \cdot 49 \\
3 \cdot 1 \\
46 \\
17 \\
20\end{array}$ & $\begin{array}{l}1869 \\
54 \\
9 \\
6 \\
43 \\
29 \\
23 \\
14 \\
14 \\
57 \\
54 \\
7 \cdot 24 \\
9 \\
6 \\
26 \\
11 \\
3 \\
6 \\
246 \\
109 \\
0 \cdot 52 \\
2 \cdot 5 \\
34 \\
14 \\
0\end{array}$ & 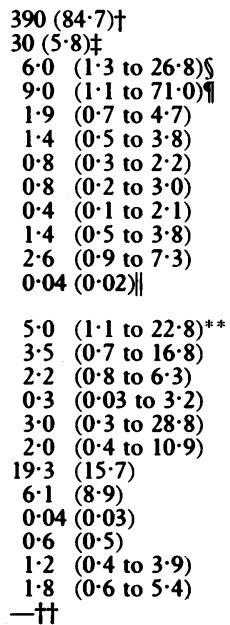 \\
\hline
\end{tabular}

* For continuous variables the mean difference and standard error is given and for dichotomous variables the odds ratio and $95 \%$ confidence intervals are presented. $p$ Values were obtained using McNemar's test for the dichotomous variables, paired $t$ test for birthweight centile data, and Wilcoxon rank sign test for other continuous variables.

$\nmid p<0.001, \neq p<0.001, \oint p=0.002, \uparrow p=0.03, \| p=0.05,{ }^{* *} p=0.04,+t p=0.02$. lower than the controls $(\mathrm{p}<0.001)$ and there was an appreciable increase in the proportion of babies with NEC with significant growth retardation ( $<10$ th centile: $p=0.02, \quad O R=6.0$, $\mathrm{CI}=1 \cdot 3$ to $26 \cdot 8 ;<3$ rd centile: $\mathrm{p}=0 \cdot 03, \mathrm{OR}=9 \cdot 0$, $\mathrm{CI}=1 \cdot 1$ to $71 \cdot 0)$. In contrast to the group of 25-29 weeks' gestation, babies with NEC in this group when compared with controls had significantly lower arterial cord $\mathrm{pH}(\mathrm{p}=0.05)$ and Apgar scores at 1 minute $(p=0 \cdot 04, O R=5 \cdot 0$, $\mathrm{CI}=1 \cdot 1$ to $22 \cdot 8$ ), but did not show a significant difference with regard to feeds.

\section{TERM INFANTS}

The eight term infants who developed NEC had a mean (SD) birth weight of $3121(469) \mathrm{g}$, mean birthweight centile of 46 (33), and gestational age of 38 (1) weeks. In four the onset of NEC followed shortly after an exchange transfusion (three via an umbilical venous catheter and one via peripheral lines) and were previously well term infants. In two, there was profound birth asphyxia (cord pH 6.96 and 6.97) while in the seventh the baby was shocked at birth due to fetomaternal transfusion, with an initial packed cell volume of $0 \cdot 15$. In the eighth the mother abused multiple drugs including heroin and cocaine, and the baby was polycythaemic and hypoglycaemic after delivery.

GROUP WITH SPONTANEOUS PERFORATION

There were six infants who developed pneumoperitoneum, but who had neither pneumotosis on radiography nor pathological findings on laparotomy or postmortem specimens consistent with NEC, and were therefore excluded as cases of NEC. They were more immature than the babies with NEC (mean gestational age 26 (2) weeks). Two of the six were never fed and only one had birth asphyxia. The presumed mechanism in these cases is perforation in an area of focal ischaemia, ${ }^{16}$ perhaps due to emboli.

\section{Discussion}

This study includes one of the largest group of babies with NEC that have been analysed using a matched case-control design. It also has the advantage that the babies were all managed in a single nursery from birth. Matching for gestational age has allowed insights into NEC that have been difficult to appreciate in previous studies matching for birth weight or with no matching at all. The results show a definite pattern of changing risk factors dependent on the gestational age. The principal factor is prematurity itself, as demonstrated in fig 1 where the incidence was $10 \%$ for 25 week infants, falling to $0.03 \%$ at term.

The group with gestational age of 25-29 weeks is notable for the lack of identifiable risk factors. An elegant model to possibly explain the occurrence of NEC in this group of infants has been proposed by Clark and Miller. ${ }^{20}$ Their model involves the pathological interaction of milk constituents and bacteria on the distal gut mucosa, and is independent of perinatal risk factors. The only perinatal factor that was related significantly to the incidence of NEC 
was whether or not the infant was given expressed breast milk. Our finding of a protective effect of expressed breast milk is in agreement with the recent report of Lucas and Cole. ${ }^{6}$ Such a protective effect may have been missed in previous studies because of small numbers of babies who received breast milk, or failure to control for gestational age. For example, in two studies the babies receiving expressed breast milk had a lower mean gestational age than those who received formula. ${ }^{21} 22$ Hence, as can be seen from fig 1, they would be expected to have an increased incidence of NEC that may have offset any improvement due to the expressed breast milk. The finding of a protective effect caused by the expressed breast milk is further supported by the prospective study of Eibl et al in which an oral immunoglobulin $\mathrm{A}$ /immunoglobulin $\mathrm{G}$ mixture was found to significantly reduce the incidence of NEC. ${ }^{23}$

It can be seen from table 2 that babies with NEC in the 30-36 week range are significantly more growth retarded than controls, and had a higher incidence of asphyxia, as evidenced by lower 1 minute Apgar scores and cord $\mathrm{pH}$. There were no other significant differences. The recent reports of an association between AREDF in the fetal umbilical artery or aorta and the subsequent development of NEC raises the questions of whether the association between NEC and growth retardation found in this study is primary, or secondary to a high proportion of these infants having AREDF. As Doppler flow studies have been performed only on selected fetuses during the last two years at this hospital, there are not enough data at present to address this question.

It is interesting that a significant association between NEC and growth retardation was not reported in the study of Stoll et al who reported $46 \%$ of their babies with NEC to be small for gestational age (SGA). ${ }^{5}$ However, $38 \%$ of their controls, matched for birth weight, were also reported as being SGA, and comparison of these groups failed to yield growth retardation as a significant difference. Similarly, the association was not reported by Kleigman et al despite the fact that $27 \%$ of their cases of NEC were below the 3 rd centile. ${ }^{4}$ However, by choosing babies of $<1500 \mathrm{~g}$, a control population resulted which included an excess of SGA babies at the higher gestations, and $22 \%$ of the 553 babies without NEC were less than the 3rd centile. As a consequence, comparison of these two populations again failed to yield growth retardation as a risk factor.

NEC in term babies is a rare event $(0.03 \%$ incidence in this series). The eight babies described here had well defined events that could compromise the gut in each case. The findings of a relationship between NEC and exchange transfusion, asphyxia, shock, or polycythaemia in more mature babies is well recognised. ${ }^{24-28}$ It is difficult not to implicate ischaemia in the pathogenesis of NEC in this group.

In summary, our results support a model of NEC in which susceptibility to NEC is inversely related to gestational age. Term infants have relatively mature gastrointestinal and immune systems, and require a significant insult before NEC can develop, whereas in babies $<30$ weeks' gestation NEC occurs most commonly in the absence of a well defined insult, the primary risk being gut immaturity itself. Between 30 weeks and term, factors such as growth retardation and asphyxia become increasingly significant. We conclude that babies at risk of NEC include all babies $<30$ weeks, all growth retarded babies (especially those with AREDF), and term babies with severe asphyxia, shock, exchange transfusion, or polycythaemia. Our findings also support the view that breast milk is associated with a reduced risk of NEC, especially in babies of 25-29 weeks' gestation.

1 Covert RF, Neu J, Elliot MJ, Rea JL, Gimotty PA. Factors associated with age of onset of necrotizing enterocolitis. $A m$ f Perinatol 1989;6:455-60.

2 De Curtis M, Paone C, Vetrano G, Romano G, Paludetto R, Ciccimarra F. A case-control study of necrotising enterocolitis occurring over 8 years in a neonatal intensive care unit. Eur $\mathcal{F}$ Pediatr 1987;146:398-400.

3 Kanto WP Jr, Wilson R, Breart GL, et al. Perinatal events and necrotizing enterocolitis in premature infants. $A m \mathcal{F}$ Dis Child 1987;141:167-9.

4 Kliegman RM, Hack M, Jones P, Fanaroff AA. Epidemiologic study of necrotizing enterocolitis among low-birthweight infants. $P$ Pediatr 1982:100:440 4 .

5 Stoll BJ, Kanto WP, Glass RI, Nahmias AJ, Brann AW Epidemiology of necrotizing enterocolitis: a case control Epidemiology of necrotizing entero
study. F Pediatr 1980;96:447-51.

6 Lucas A, Cole TJ. Breast milk and neonatal necrotising enterocolitis. Lancet 1990;336:1519-23.

7 Palmer SR, Thomas SJ, Cooke RW, et al. Birthweight specific risk factors for necrotising enterocolitis. I Epidemiol Community Health 1987;41:210-4.

8 Smith MF, Borriello SP, Clayden GS, Casewell MW. Clinical and bacteriological findings in necrotising enterocolitis: a controlled study. F Infect 1980;2:23-31.

9 Yu VY, Tudehope DI. Neonatal necrotising enterocolitis: 2. Perinatal risk factors. Med $\mathcal{J}$ A ust 1977; i:688-93.

10 Bunton GL, Durbin GM, McIntosh N, et al. Necrotising enterocolitis. Controlled study of 3 years' experience in a neonatal intensive care unit. Arch Dis Child 1977;52:772-7.

11 Yu VY, Joseph R, Bajuk B, Orgill A, Astbury J. Perinatal risk factors for necrotising enterocolitis. Arch Dis Child 1984:59:430-4

12 Wilson R, Kanto WP, McCarthy BJ, Feldman RA. Age at onset of necrotizing enterocolitis: risk factors in small infants. Am $\mathcal{A}$ Dis Child 1982;136:814-6.

13 Vincour P, Stine MJ. Risk factors for late-onset necrotizing enterocolitis. Indiana Med 1990; July:478-80.

14 Malcolm G, Ellwood D, Devondale K, Beilby R, HendersonSmart $D$. Absent or reversed end diastolic flow velocity in the umbilical artery and necrotising enterocolitis: a casecontrol study. Arch Dis Child 1991;66:805-7.

15 Hackett GA, Campbell S, Gamsu H, Cohen-Overbeek T, Pearce JM. Doppler studies in the growth retarded fetus and prediction of neonatal necrotising enterocolitis, hae morrhage, and neonatal morbidity. BMF 1987;294:13-6.

16 Weinberg G, Kleinhaus S, Boley SJ. Idiopathic intestin perforation in the newborn: an increasingly common entity. f Pediatr Surg 1989;24:1007-8.

17 Clinical Reporting Systems. CRS manual. Castle Hill, Australia: CRS Pty Ltd, 1991.

18 SPSS. SPSS/PC + V2.0; Statistical package for the socia sciences. Chicago: SPSS Inc, 1990

19 Dean AG, Dean JA, Burton AH, Dicker RC. EPI INFO, version 5: a word processing, database and statistics program for epidemiology on microcomputers. Stone Mountain, Georgia: USD, 1990.

20 Clark DA, Miller MJ. Intraluminal pathogenesis of necrotizing enterocolitis. F Pediatr 1990;117:S64-7.

21 Kliegman RM, Pittard WB, Fanaroff AA. Necrotizing enterocolitis in neonates fed human milk. $\mathcal{I}$ Pedia enterocolitis in

22 Moriartey RR, Finer NN, Cox SF, et al. Necrotizing enterocolitis and human milk. F Pediatr 1979;95:295-6.

23 Eibl MM, Wolf HM, Furnkranz H, Rosenkranz A. Prevention of necrotising enterocolitis in low birthweight infants by IgA-IgG feeding. $N$ Engl F Med 1988;319:1-7.

24 Kliegman RM, Fanaroff AA. Neonatal necrotizing enterocolitis: a nine year experience. Epidemiology and uncommo observations. Am $\mathcal{F}$ Dis Child 1981;135:603-7.

25 Yu VY, Tudehope DI, Gill GJ. Neonatal necrotising enterocolitis: 1 . Clinical aspects. Med $\mathcal{F}$ A ust 1977;i:685-8.

26 Hakanson DO, Oh W. Necrotizing enterocolitis and hyperviscosity in the newborn infant. $\mathcal{F}$ Pediatr 1977;90:458-61.

27 Wilson R del Portillo M, Schmidt E, Feldman RA, Kanto WP. Risk factors for necrotizing enterocolitis in infants weighing more than 2000 grams at birth: a case-control study. Pediatrics 1983;71:19-22.

28 Polin RA, Pollack PF, Barlow B, et al. Necrotizing enterocolitis in term infants. $\mathcal{F}$ Pediatr 1976;89:460-2. 